\title{
AVALIAÇÃO DOS SERVIÇOS DE SAÚDE NA ÓTICA DOS USUÁRIOS
}

\section{Lucimare Ferraz}

Universidade Comunitária da Região de Chapecó (UNOCHAPECÓ)

\section{Maia Elizabeth Kleba}

Universidade Comunitária da Região de Chapecó (UNOCHAPECÓ)

Fátima Ferretti

Universidade Comunitária da Região de Chapecó (UNOCHAPECÓ)

\section{Natacha Luana Pezzuol Frank}

Universidade Comunitária da Região de Chapecó (UNOCHAPECÓ)

\section{AVALIAÇÃO DOS SERVIÇOS DE SAÚDE NA ÓTICA DOS USUÁRIOS}

Resumo: A avaliação é uma importante ferramenta da atenção básica, devendo subsidiar a organização e orientação dos serviços de saúde, a partir de lógicas mais centradas no usuário. Este artigo tem por objetivo apresentar a ótica dos usuários sobre os serviços de saúde inseridos no território de um núcleo de apoio à saúde da família. Os dados provêm de um estudo quantitativo-descritivo desenvolvido com 1023 famílias, cadastradas pela Estratégia Saúde da Família. Os resultados revelaram que $89,3 \%$ das famílias utilizam serviços do centro de saúde, tendo como principal motivo a busca por assistência em situação de doença ou dor $(76,3 \%)$. Já no que tange a resolutividade, somente $9,1 \%$ relataram que quase nunca têm seu problema resolvido e $51,1 \%$ consideram bom o atendimento no centro de saúde. O artigo destaca, ainda, a satisfação do usuário como dimensão relevante na avaliação da atenção à saúde para o aprimoramento dos serviços e das políticas públicas em saúde.

Palavras-chave: Avaliação de Serviços de Saúde, Atenção Primária à Saúde, Satisfação do Paciente.

\section{EVALUATION OF HEALTH SERVICES IN THE USERS' VIEW}

Abstract: The evaluation is an important tool of the primary care that should subsidize the organization and orientation of health services, from logics more centered in the user. This article aims to present the users' perspective about health services included in the territory of a core of support for family health. The data comes from a quantitative-descriptive study developed with 1023 families registered by the Family Health Strategy. The results revealed that $89,3 \%$ of families use services from the health center, having as it's main reason the searching for assistance in situations of illness or pain $(76,3 \%)$. Regarding the resolution, only $9,1 \%$ reported that they almost never have their problem solved and $51,1 \%$ considered good the care at the health center. The article still highlights the user satisfaction as a relevant dimension in the evaluation of health care for the improvement of services and public health policies.

Keywords: Evaluation of Health Services, Primary Attention to Health, Patient Satisfaction. 


\section{INTRODUÇÃO}

Em 1986, na $8^{a}$ Conferência Nacional de Saúde, foram lançados os fundamentos para a criação do Sistema Único de Saúde (SUS), instituído,em 1988, como um conjunto de ações e serviços que visam, entre outros, prestar assistência integral às pessoas por meio de ações de promoção, proteção e recuperação da saúde. O SUS possui como alicerce o conceito global de saúde e a garantia de igualdade e universalidade de acesso aos serviços de saúde (MENDES, 1999). Para orientar a reorganização da Atenção Básica, surge em 1994 a Estratégia Saúde da Família (ESF), denominada inicialmente de Programa Saúde da Família (PSF), como modelo de assistência que visa favorecer a reorientação do processo de trabalho de acordo com os preceitos do SUS, ampliando a resolutividade e o impacto na situação de saúde das pessoas e coletividades, no nível de atenção primária (BRASIL, 1994; BRASIL, 2011a).

Com a implantação da ESF, o Ministério da Saúde buscou viabilizar uma organização dos serviços e ações em saúde mais coerente com o conceito ampliado da saúde, com o objetivo de substituir o clássico modelo assistencial, que tinha como foco o cuidado hospitalar, meramente curativo, individualizante e médico-centrado, implicando em alto custo para o sistema de saúde e baixa resolutividade (VASCONCELOS, 2000). A ESF tornou-se, desde então, a porta de entrada preferencial para a Atenção Básica (AB), com o estabelecimento de equipes multiprofissionais para atuar na unidade de saúde e na comunidade, responsáveis por um número determinado de famílias localizadas num território (BRASIL, 2012b).

Desde sua criação, a cobertura da ESF vem se expandindo expressivamente. Segundo dados mais recentes do Departamento de Atenção Básica, até o ano de 2009 já haviam sido implantadas 30.328 equipes de saúde da família, o que correspondia a $50,7 \%$ da população brasileira atendida pela estratégia, cerca de 96,1 milhões de pessoas. Este avanço na assistência à saúde tem contribuído com a produção de resultados positivos nos principais indicadores de saúde, melhorando a qualidade de vida das famílias assistidas (BRASIL, 2012c). A priorização e o maior investimento público na ESF justificam a necessidade de se realizar estudos que revelem seu potencial na efetivação dos princípios do SUS.

Para melhorar esses indicadores foram criados em 2008 os Núcleos de Apoio à Saúde da Família (NASF) com o objetivo de ampliar os serviços da atenção básica e de incorporar à Equipe de Saúde da Família profissionais de diferentes áreas do conhecimento, tais como assistente social, educador físico, fisioterapeuta, nutricionista, psicólogo, terapeuta ocupacional, entre outros.

Ressalta-se que o NASF não se constitui porta de entrada do sistema para os usuários, mas sim de apoio às equipes de Saúde da Família. Os processos de trabalho do NASF devem ter como foco a ampliação das ações da Atenção Básica, no território sob sua responsabilidade, priorizando o atendimento compartilhado e interdisciplinar, com troca de saberes, capacitação e responsabilidades mútuas, para incrementar não só a integralidade, mas também a resolutividade e a qualidade do cuidado (BRASIL, 2010).

Considerando que há um amplo debate sobre a necessidade de atender os princípios do SUS e que os estudos avaliativos são importantes ferramentas para detectar informações que nos permitam replanejar as ações com vistas a melhorar as práticas de atenção e, que a opinião do usuário é fundamental nesse processo, o presente estudo, teve por objetivo apresentar a ótica avaliativa dos usuários sobre os serviços de saúde desenvolvidos pela atenção básica no território de um Núcleo de Apoio à Saúde da Família (NASF), na perspectiva de ampliar o debate sobre as contribuições da ESF na qualificação do serviço e na resolutividade das políticas de saúde.

\section{AVALIAÇÃO DE SERVIÇOS DE SAÚDE}

A definição do termo avaliação de políticas, programas e ações não possui um consenso e, tem sido utilizada sob várias perspectivas. Para Patton (1997), a avaliação pode ser designada como uma atividade focada para utilização, devendo-se levar em consideração o modo de execução, com vistas à orientação e reorganização das ações. Nessa perspectiva, Contandriopoulos (et al. 1997) compreende a avaliação como o julgamento sobre a forma de conduzir as ações, e também as intervenções feitas. A avaliação de um programa justifica-se para se ter conhecimento acerca da real situação encontrada, se os objetivos inicialmente propostos foram alcançados, além de servir como base para a reorientação de mudanças no serviço (McLAUGHIN; JORDAN, 1999).

No contexto da avaliação em saúde, muitos são os desafios encontrados. Inicialmente por esta área ser de natureza complexa e receber influências de vários fatores, o que dificulta a delimitação dos objetos a serem ava- 
liados, além de conter uma porção subjetiva referente aos componentes relacionais, o que interfere na definição de consensos. Ainda pode-se apontar como desafio, a necessidade de contextualização para a aplicação da pesquisa, sendo inevitável a abrangência das particularidades de cada programa. Isso justifica a necessidade e a relevância de instituir processos de avaliação permanente dos sistemas de saúde, considerando especificidades locais e a perspectiva dos sujeitos envolvidos, para aprimorar o serviço ofertado e promover melhorias na qualidade de vida dos usuários (MEDINA et al., 2005).

As iniciativas de avaliação, tomando como matéria-prima a perspectiva do usuário, difundiu-se a partir dos anos 60 na Europa e nos EUA com um foco inicial na adesão ao tratamento. Nos anos de 1970 e 1980, essa prática passou a ser incorporada na avaliação da qualidade dos serviços de saúde, ganhando, no Brasil, destaque a partir da segunda metade da década de 90 (GOMES et al., 2011).

Entre os estudiosos desta temática, Donabedian (1978) produziu importantes publicações na área de avaliação da qualidade dos serviços de saúde. Segundo ele, o objetivo da avaliação da qualidade é estabelecer o nível de êxito das profissões na área da saúde em se autogovernarem, para assim evitar o abuso ou a incompetência. Por outro lado, o objetivo da monitorização da qualidade é exercitar a supervisão constante, para que, quando surgirem anormalidades, as reparações sejam imediatas. Tal avaliação dos serviços abrange duas dimensões: a primeira o desempenho técnico, que se caracteriza pelo emprego do conhecimento e tecnologia médica, para o bem-estar do paciente. A segunda, a relação pessoal com o paciente, respeitando preceitos éticos, desejos e necessidades do paciente, que implica em elementos comportamentais.

No Brasil, a fundamentação do objetivo de avaliar o Sistema Único de Saúde (SUS) baseia-se em dar suporte às decisões que serão tomadas, reconhecer os problemas e conduzir os serviços e ações a serem desenvolvidos, além de verificar o impacto do serviço de saúde sobre a população (BRASIL, 2005; BRASIL, 2011a). Nessa perspectiva, "institucionalizar a avaliação deve ter sentido de integrá-la em um sistema organizacional [...] ligando necessariamente as atividades analíticas às de gestão das intervenções programáticas" (HARTZ, 2002, p. 419), lembrando que, na saúde, a avaliação visa melhorar as práticas de atenção e gerar processos condizentes com os preceitos do SUS, como equidade, resolutividade, universalidade, integralidade, acessibilidade e participação social (FELISBERTO, 2004).

Para o Ministério da Saúde (MS), a institucionalização da avaliação precisa estar associada à formação de uma política de avaliação no contexto do SUS, na qual a Atenção Básica $(A B)$ deve estar inclusa neste processo, englobando todas as pessoas envolvidas (usuários, gestores, profissionais de saúde, pesquisadores), suas respectivas funções e método de financiamento (BRASIL, 2005). A avaliação na $A B$, e mais especificamente na $E S F$, é referida como importante recurso de reconhecimento das necessidades de organização e reorientação das ações e serviços por lógicas mais centradas no usuário. Cabe a toda equipe da ESF, participar do acolhimento dos usuários, realizando a escuta qualificada de suas necessidades em saúde para proporcionar atendimento humanizado e viabilizar o estabelecimento do vínculo. O MS refere vínculo como relações de afetividade e confiança, construídas entre usuário e trabalhador, que permitem aprofundar o processo de corresponsabilização pela saúde (BRASIL, 2011a).

No entanto, apesar dos avanços na metodologia dos processos avaliativos, observa-se que a opinião do usuário sobre o sistema de saúde não tem sido valorada devidamente. Sem dúvida, o usuário dos serviços de saúde é um avaliador em potencial, pois é o principal envolvido nas atividades desenvolvidas pela equipe da ESF e vivencia o cotidiano dos serviços quando procura pelo atendimento na unidade de saúde. A avaliação na área da saúde não deve ser apenas o monitoramento das ações e normas, mas também uma forma democrática de empoderamento dos cidadãos na confirmação de seus direitos, pois o usuário caracteriza-se como mais um dos atores corresponsáveis. Este direito confere ao cidadão a participação na tomada de decisões, sendo importante ter cuidado em relação à diversidade dos sujeitos e suas respectivas especificidades, de forma a incluir desejos e necessidades expressadas por cada um dos atores envolvidos (PINHEIRO; MARTINS, 2011). O caráter subjetivo da avaliação agrega diferentes sentimentos e emoções, delimitando, assim, as expectativas dos usuários. Quando busca uma avaliação centrada na integralidade do cuidado e prevenção, a participação comunitária no processo avaliativo é fundamental (AYRES, 2004).

A atividade conjunta de todos os atores do sistema de saúde (profissionais de saúde, gestores, técnicos e usuários) fortifica a rede social, ampliando o cuidado em saúde e diminuin- 
do o sofrimento e adoecimento dos usuários. Assim, acredita-se que, a maior comunicação entre os atores nas instituições de saúde, meIhora o acolhimento e supressão das necessidades da população assistida, adequando assim, o oferecimento de serviços voltados à demanda existente.

Como destaca Gomes (et al. 2011), a opinião do usuário para além de constituir-se como indicador sensível da qualidade do serviço prestado é uma categoria considerada estratégica para uma maior adequação dos serviços às suas demandas, fator fundamental para a efetivação dos princípios do SUS.

\section{METODOLOGIA}

O estudo teve uma abordagem de caráter exploratório-descritivo com delineamento de um estudo transversal, sendo desenvolvido durante o primeiro semestre de 2011, no território de um dos Núcleos de Apoio à Saúde da Família (NASF) do município de Chapecó/SC. Esse território foi definido, em diálogo com o gestor municipal, como espaço prioritário para desenvolvimento dos projetos aprovados em 2008 pela Unochapecó em parceria com a Secretaria da Saúde do município no Programa Nacional de Reorientação da Formação Profissional em Saúde (Pró-Saúde).

A população do estudo foi constituída por famílias cadastradas pelos centros de saúde inseridos no referido NASF, num total de 13.866, de acordo com os dados do SIAB de fevereiro de 2011. A amostra selecionada foi de 1.133 famílias, com erro amostral de $3 \%$ e proporção de perda em $10 \%$, das quais 1.023 foram efetivamente incluídas.

Os domicílios foram selecionados aleatoriamente por meio das fichas A dos cadastros dos agentes comunitários de saúde (ACS) pertencentes aos centros de saúde já referidos. Num primeiro momento foram reunidos os prontuários das famílias de acordo com sua microárea de residência, sendo então todos os prontuários agrupados em uma única pilha; em seguida foi selecionada a oitava família (número sorteado pela equipe de pesquisadores) e, a partir dessa, as próximas subsequentes com intervalo de 12 prontuários, até atingir a valor da amostra determinado para cada centro de saúde. Na terceira etapa, houve a confirmação com o ACS responsável pela família, se a mesma ainda residia no endereço registrado e se havia restrição de horário para a visita/entrevista, procedendo-se, neste caso, sorteio de nova família.

A coleta de dados foi realizada por meio de um questionário estruturado com perguntas sobre uso e acesso aos serviços de saúde, entre outros. O questionário foi construído com a participação de profissionais ligados à gestão da saúde, em especial a gerência da Atenção Básica, buscando contemplar questões que pudessem contribuir com a qualificação do planejamento das equipes da ESF do município.

A equipe de trabalho foi composta por 108 acadêmicos, 48 profissionais da saúde (preceptores da Secretaria da Saúde de Chapecó) e 14 docentes dos programas Pró-Saúde, Pet-Saúde e Pet-Visa ${ }^{1}$ (12 tutores da Unochapecó, e dois coordenadores dos programas). Os acadêmicos foram os principais coletores dos dados, supervisionados pelos profissionais de saúde. A análise dos dados ficou sob a responsabilidade dos docentes dos programas sob a supervisão das coordenadoras da pesquisa.

Para o tratamento dos dados foram utilizadas técnicas próprias para um estudo descritivo, as quais informam sobre as médias e a frequência (absoluta e relativa) da distribuição de um determinado evento na população em estudo. Os dados coletados foram codificados, tabulados e digitados em bancos de dados, especialmente construídos no programa Statistical Package for Social Science (SPSS). Após a digitação, os dados sofreram o processo de controle de qualidade com análise de coerência e consistência. Após a correção de erros de digitação procedeu-se a análise estatística dos dados.

Com relação aos aspectos éticos da pesquisa, o projeto foi aprovado pelo Comitê de Ética em Pesquisa da Universidade Comunitária da Região de Chapecó sob Parecer 001/2011.

\section{RESULTADOS E DISCUSSÃO}

O município de Chapecó, situado na região oeste de Santa Catarina, conta atualmente com aproximadamente 180 mil habitantes. Em sua rede de saúde, dispõe de 26 Centros de Saúde, com um total de 32 equipes da ESF, o que corresponde a mais de $75 \%$ de cobertura da população. Em 2010, o município instituiu quatro Núcleos de Apoio à Saúde da Família (NASF), sendo que o selecionado para este estudo integra quatro Centros de Saúde, somando oito equipes da ESF.

Neste estudo, constatou-se que $89,1 \%$ $(n=911)$ das 1.023 famílias entrevistas utilizam os serviços do centro de saúde do bairro, demonstrando uma cobertura da ESF acima das médias nacional. Entre as famílias do estudo que possuem plano de saúde $(31,2 \%)$, destas, $20,7 \%$ utilizam ainda o serviço de saúde públi- 
co (Unidade Básica de Saúde) do seu território de moradia. Estes números podem indicar, por um lado, limitações relativas a oferta e/ou ao acesso ao serviços dos planos de saúde e, por outro lado, maior facilidade e/ou acesso aos serviços públicos no município.

Conforme dados da Agência Nacional de Saúde Suplementar, até setembro de 2012 o índice de abrangência da população brasileira beneficiária de planos privados de saúde é de $25,1 \%$ (BRASIL, 2012a). A pesquisa realizada por Turrini, Lebrão e Cesar (2008) em cinco municípios do estado de São Paulo, por meio de inquérito domiciliar para população atendida pela ESF, encontrou prevalência de $64,1 \%$ dos entrevistados como dependentes do sistema público de saúde, e $35,5 \%$ possuíam plano privado de saúde. Santos (1995) ressalta a questão econômica como forma determinante para a escolha entre o serviço de saúde público e o privado, mas afirma que o atendimento médico é semelhante em ambos os serviços, tendo como variantes a rapidez e agilidade no acesso ao serviço privado. Ainda aponta que a oferta de medicamentos sem custo adicional na rede pública é um fator que determina a escolha por este serviço, principalmente, por aqueles que possuem dificuldades financeiras para sua aquisição.

No estudo realizado verificou-se que em $76,3 \%$ das famílias, algum membro procurou o centro de saúde nos três meses anteriores à pesquisa por algum problema de saúde, sendo apontado, como principal motivo, (apontado) a doença ou a dor, e em segundo lugar realização de consulta de rotina, como mostra a tabela 1.

TABELA 1: Motivo principal da procura pelo atendimento em saúde dos integrantes da família nos últimos três meses (a partir de novembro de 2010), Chapecó-SC, 2011.

\begin{tabular}{lcc}
\hline $\begin{array}{l}\text { Motivo pela procura de aten- } \\
\quad \text { dimento em saúde }\end{array}$ & $\mathrm{N}$ & $\%$ \\
\hline Doença ou dor & 459 & 58,8 \\
Para fazerconsulta de rotina & 165 & 21,1 \\
Atendimentos preventivos & 42 & 5,3 \\
Atendimento enfermagem & 41 & 5,2 \\
Atendimento odontológico & 23 & 3,0 \\
Pré-natal & 20 & 2,5 \\
Acidente ou lesão & 20 & 2,5 \\
Atestado de saúde & 11 & 1,4 \\
\hline Total & 781 & 100,0 \\
\hline
\end{tabular}

Fonte: elaboração própria a partir do relatório da Agência Nacional de Saúde Suplementar (2012)
De acordo com Siméant (1984), existem dois tipos de demanda na atenção básica: a espontânea, que surge conforme necessidades constatadas, e a induzida, que resulta da mobilização do Estado por meio de programas preventivos de saúde. No estudo realizado evidenciou-se que a maioria das famílias corresponde à demanda espontânea na procura por atendimento na UBS.

Em estudo realizado por Paiva, Bersusa e Escuder (2006) em uma UBS para avaliar o serviço de saúde ofertado sob a percepção do usuário, notou-se que pacientes com doenças crônicas degenerativas necessitam de maior investimento do serviço de saúde, principalmente no âmbito de ações preventivas e de promoção à saúde, e como há um aumento da demanda por essas doenças, sugere-se que a atenção básica faça uma adequação da oferta do serviço a esta população para abranger suas necessidades de saúde.

Alguns fatores influenciam a utilização dos serviços públicos de saúde. Para Turrini, Lebrão e Cesar (2008), a busca por atendimento no sistema de saúde depende do acesso e do acolhimento dos usuários, do número de serviços ofertados e da disponibilidade de profissionais, da necessidade do usuário de ausentarse do trabalho para receber atendimento, de questões financeiras, sendo ainda relevante a concepção do indivíduo sobre o processo saúde-doença. Travassos et al. (2000) reiteram estes fatores, e acrescentam o cenário geográfico, a cultura e a visão dos usuários sobre o sistema como fatores relevantes.

Quanto às dificuldades vivenciadas pelos integrantes da família no que tange à procura pela assistência, observa-se que $60,9 \%$ relataram não ter nenhum tipo de problema em buscar os serviços de saúde. Entre aqueles que mencionaram dificuldades, $40,17 \%$, destacase a crença na indisponibilidade de consulta médica como o fator mais limitante, e a dificuldade financeira foi mencionada em 23,93\%. Diante do cenário encontrado na pesquisa sobre o índice de famílias que acreditavam não ter consulta acessível, vale ressaltar que o Ministério da Saúde estabelece como parâmetros para a cobertura da atenção básica duas formas para organizar a procura por atendimento médico: o agendamento e a demanda espontânea, distribuindo a assistência médica em 16 consultas por período de quatro horas, sendo 12 para agendamento e quatro para livre demanda (BRASIL, 2002).

Puccini e Cornetta (2008), com o objetivo de avaliar o serviço da atenção básica por meio de evento sentinela - que na área da saúde 
identifica um fato que não deveria acontecer se o funcionamento da atenção básica fosse adequado, resultando em falha deste nível de atenção -, analisaram quatro Unidades Básicas de Saúde (UBS) cuja população fazia uso do Pronto-Socorro da cidade como serviço de referência e os resultados obtidos sobre o motivo pelo qual a UBS não foi procurada, apontaram que $47,8 \%$ dos entrevistados tiveram dificuldade em conseguir vaga no dia, $11,5 \%$ mencionaram a falta de médicos, e $7,1 \%$ o problema na obtenção de agendamento das consultas. Em outro estudo, Frez e Nobre (2011) também identificaram como principal problema no que tange ao acesso à atenção básica, a dificuldade em marcar consultas.

A Organização Mundial da Saúde preconiza que a atenção básica deve exercer papel de porta de entrada do sistema de saúde, possuindo capacidade para resolver pelo menos $80 \%$ dos agravos à saúde da comunidade abrangente (MADUREIRA; DE CAPITANI; CAMPOS, 1989). A habilidade resolutiva varia conforme a quantidade e distribuição de profissionais para absorverem a demanda encontrada. Esta é avaliada pelo número de usuários acolhidos sobre aqueles que solicitaram atendimento, sendo o critério de mensuração o porte de demanda reprimida (SIMÉANT, 1984).

Por outro lado, a resolutividade não se restringe à quantidade de consultas oferecidas ou ao acesso a consultas médicas, mas abrange o conjunto de ações desenvolvidas pela equipe no sentido de oferecer respostas adequadas e satisfatórias aos problemas de saúde referidos pelos usuários e identificados em diagnósticos mais abrangentes junto à comunidade. Nesse sentido, a Portaria da Atenção Básica (2011) refere entre suas funções, a de:

[...] coordenar o cuidado: elaborar, acompanhar e gerir projetos terapêuticos singulares, bem como acompanhar e organizar o fluxo dos usuários entre os pontos de atenção das RAS. Atuando como o centro de comunicação entre os diversos pontos de atenção, responsabilizando-se pelo cuidado dos usuários em qualquer destes pontos [...] com o objetivo de produzir a gestão compartilhada da atenção integral. Articulando também as outras estruturas das redes de saúde e intersetoriais, públicas, comunitárias e sociais. Para isso, é necessário incorporar ferramentas e dispositivos de gestão do cuidado, tais como: gestão das listas de espera (encaminhamentos para consultas especializadas, procedimentos e ex- ames), prontuário eletrônico em rede, protocolos de atenção organizados sob a lógica de linhas de cuidado [...] (BRASIL, 2011b, p. 4).

Em nosso estudo, os membros das famílias quando questionados sobre a resolução dos problemas de saúde após a procura pelo atendimento no centro de saúde do seu bairro, a maioria relatou que seus problemas foram resolvidos, como mostra a tabela 2 .

TABELA 2: Resolutividade autopercebida pelas famílias sobre o atendimento na unidade de saúde, Chapecó-SC, 2011.

\begin{tabular}{lcc}
\hline Resolução do problema de saúde & $\mathrm{N}$ & $\%$ \\
\hline Sempre/quase sempre & 541 & 52,9 \\
Ás vezes & 317 & 31,0 \\
Quase nunca & 93 & 9,1 \\
Não se aplica & 58 & 5,7 \\
lgnorado & 14 & 1,4 \\
\hline Total & 1023 & 100,0 \\
\hline $\begin{array}{l}\text { FONTE: elaboração própria a partir do relatório da Agência Na- } \\
\text { cional de Saúde Suplementar (2012). }\end{array}$ &
\end{tabular}

Em um estudo executado por Medeiros et al. (2010) em uma UBS de um município de médio porte no Brasil, o índice de satisfação dos usuários do sistema de saúde quanto à resolutividade foi de $79,2 \%$. Já noutra pesquisa realizada no estado do Rio Grande do Sul, a percentagem da resolutividade apontada por usuários da ESF foi de somente $34,4 \%$ sempre que estes utilizavam o serviço, $26,9 \%$ na maioria das vezes, e $32,3 \%$ às vezes (ROSA; PELEGRINI; LIMA, 2011).

Por outro lado, a pesquisa produzida em cinco municípios do estado de São Paulo por Turrini, Lebrão e Cesar (2008), através de inquérito domiciliar para população atendida pela ESF, encontrou uma taxa de resolutividade do serviço de 97,8\%. Essas disparidades de resultados demonstram a necessidade de se instituir uma cultura de avaliação nos serviços de saúde, em que haja uma maior padronização dos modelos e protocolos, para que assim, tenha-se mais informações sobre a satisfação e resolutividade dos serviços e políticas de saúde, pois não se concebe hoje um trabalho sério de cuidados à saúde sem esse diagnóstico, que envolve a participação popular, o diálogo intersetorial e a descentralização da política de saúde, a fim de planejar as ações de acordo com as condições e necessidades locais existentes.

Nesse sentido, avaliar o sistema, por meio de estudos que identifiquem a qualidade do

R. Pol. Públ., São Luís, v. 17, n.2, p. 414 - 423, jul./dez. 2013419 
serviço ofertado aos usuários, torna-se essencial para promover as mudanças necessárias. Para tanto, torna-se imprescindível consultar a população, que é um ator importante dos avanços alcançados pela ESF, mas que às vezes atua passivamente neste processo (MISHIMA et al., 2010). Nesse sentido, Costa et al. (2008) ressaltam a importância de utilizar métodos avaliativos que deem voz à comunidade, pois as contribuições e o olhar que o usuário dará sobre o sistema ou serviço serão fundamentais para compreender as dificuldades e potencialidades de efetivação das ações e políticas e contribuirão para o processo de reorganização dos serviços.

\section{A análise dos dados coletados no es-}

tudo revela que $51,1 \%$ das famílias entrevistadas consideraram oatendimento como bom, conforme apresentado na tabela 3.

TABELA 3: Avaliação do atendimento realizado no centro/posto de saúde do seu bairro segundo opiniãodasfamílias entrevistadas,Chapecó-SC,2011.

\begin{tabular}{|l|c|c|}
\hline Atendimentonocentrodesaúde & $\mathbf{N}$ & $\%$ \\
\hline Bom & 523 & 51,1 \\
\hline Regular & 311 & 30,4 \\
\hline Ruim & 122 & 11,9 \\
\hline Ignorado* & 67 & 6,6 \\
\hline Total & 1023 & 100,0 \\
\hline
\end{tabular}

* O membro respondente absteve-se de conceder a informação. FONTE: elaboração própria a partir do relatório da Agência Nacional de Saúde Suplementar (2012).

Muitas vezes, a insatisfação do usuário pode estar associada ao seu desconhecimento sobre a organização do serviço de saúde, pois ao não conhecer as normas de funcionamento da UBS, podendo gerar a ideia de atendimento inadequado (MISHIMA et al., 2010). Na pesquisa realizada por Trad et al. (2002), a satisfação do usuário perante o sistema de saúde foi correlacionada à oportunidade de acesso aos profissionais, além das atividades de saúde, de maneira diferenciada do padrão centrado na enfermidade como era o modelo biomédico. Outro fator que cabe destacar é que pelo fato de o Brasil ser um país em desenvolvimento e a obtenção de um serviço de saúde com qualidade ser uma prerrogativa de poucos, espera-se que os índices de satisfação quanto aos serviços de saúde sejam diminuídos (ESPERIDIÃO; TRAD, 2005).

No entanto, observa-se que diante do reconhecimento da atuação da ESF como uma estratégia política que norteia o cuidado voltado à família, reorientando a dinâmica da organização das ações e substituindo o clássico modelo curativo e individualizado, é possível verificar o avanço ocorrido na assistência à saúde, evidenciando pontos positivos na análise feita pelos usuários do sistema. Contudo, reforça-se a importância da constante avaliação da atenção à saúde para o aprimoramento do serviço e a melhoria da qualidade de vida das famílias.

Sobre esse aspecto, ressalta-se que a avaliação das políticas e serviços de saúde deve ser pluralista, consistindo numa abordagem coletiva, envolvendo os diversos atores parceiros da política avaliada. Nessa metodologia "um dos efeitos esperados é desenvolver nos participantes a capacidade de assimilação dos resultados da avaliação pluralista, além de aumentar a possibilidade de adoção das medidas proposta" (TINÔCO; SOUZA; BARBOSA, 2011, p. 311).

Além disso, a avaliação deve ser considerada como um recurso técnico e político pela busca de reorientação das práticas (e da promoção) em saúde. Igualmente, a avaliação deve transcender a finalidade técnica das práticas em saúde, em sua condição limitada a produção objetiva de produtos do trabalho em saúde, definindo critérios avaliativos referentes à escolha, o desejo, o projeto de felicidade dos envolvidos no processo de cuidado á saúde (AYRES, 2004).

Vale ressaltar, que a avaliação não é neutra, pois expressa percepções de mundo e objetivos distintos, sendo comum instituir-se em um fim próprio, e não como uma forma estratégica de compreender as consequências das ações públicas (TINÔCO; SOUZA; BARBOSA, 2011). Para tanto, avaliar dando voz aos atores sociais envolvidos nas ações e serviços de saúde, bem como garantir sua participação na definição das prioridades e no acompanhamento do processo avaliativo torna-se imperativo quando o objetivo é qualificar serviços com vista a promover saúde e melhorar a qualidade de vida da população.

\section{CONCLUSÃO}

Este estudo evidenciou que $89,1 \%$ das famílias utilizam os serviços do centro de saúde do bairro, demonstrando uma cobertura da ESF acima da média nacional, e que em $76,3 \%$ da família algum membro procurou o centro de saúde nos três meses anteriores à pesquisa por doença ou dor, reforçando o paradigma de que a procura pelo atendimento se dá por meio de um sintoma ou processo patológico. Ainda, $60,9 \%$ dos usuários relataram não ter problemas para buscar os serviços de saúde e que em $52,9 \%$ das situações em que procuraram 
os serviços, sempre ou quase sempre, tiveram seus problemas de saúde resolvidos. Já no que tange à satisfação das famílias em relação aos serviços de saúde, $51,1 \%$ consideraram o atendimento bom.

O estudo demonstrou satisfação da população de quatros territórios em relação aos serviços ofertados pela atenção básica, bem como evidenciou a efetividade destes pelo olhar do usuário. Ao considerar a opinião do usuário como indicador sensível da qualidade dos serviços de saúde, e que esta pode contribuir efetivamente para uma maior adequação dos serviços às demandas da população de cada território, reconhecendo-se que se torna imprescindível incorporar a avaliação na prática cotidiana do trabalho das equipes de saúde, ouvir e se fazer ouvir, passa a ser fundamental para a efetivação dos princípios do SUS.

Ao final, resgatando o objetivo desse estudo - apresentar a ótica avaliativa dos usuários sobre os serviços de saúde- sugere-se que as estratégias e ações de saúde sejam permanentemente avaliadas pelos usuários dos serviços públicos. Além disso, que o planejamento das definições de objetivos, políticas e ações em saúde necessitam ser compartilhada com a população. Para tanto, os profissionais dos serviços devem problematizar as condições de saúde e doença com a comunidade, diagnosticando coletivamente os problemas e pactuando soluções, numa gestão democrática.

Outrossim, sem temer a críticas, deve-se possibilitar a participação dos usuários no processo de avaliação das políticas e serviços de saúde, numa perceptiva de estimular o controle social comunitário e, também, promover um elo de comunicação e integração entre a população, profissionais e gestores em saúde. Portanto, acredita-se que avaliação em saúde necessita ser baseada no pluralismo, reconhecendo a complexidade dos determinantes e condicionantes do adoecimento, dos interesses (vontades) e das responsabilidades de cada um, tendo por escopo a saúde.

$\mathrm{E}$ que, o planejamento e a implementação de um processo avaliativo contínuo, realizados pelo conjunto de atores do sistema de saúde (profissionais de saúde, gestores, técnicos e usuários), de forma compartilhada, ampliarão a efetividade do cuidado em saúde e a adequação dos serviços voltados às demandas existentes.

\section{REFERÊNCIAS}

AYRES, J. R. C. M. Norma e formação: horizontes filosóficos para as práticas de avaliação no contexto da promoção da saúde. Ciência e Saúde Coletiva, Rio de Janeiro, v. 9, n. 3, p. 583-92, 2004.

BRASIL. Ministério da Saúde. Secretaria de Assistência a Saúde. Saúde da família: uma estratégia para a reorientação do modelo assistencial. Brasília, DF: Ministério da Saúde, 1994.

BRASIL. Portaria n. 1.101/GM, de 12 de junho de 2002. Estabelecendo os parâmetros de cobertura assistencial no âmbito do Sistema Único de Saúde. Brasília, DF: Ministério da Saúde, 2002.

BRASIL. Ministério da Saúde. Secretaria de Atenção à Saúde. Departamento de Atenção Básica. Coordenação de Acompanhamento e Avaliação. Avaliação na Atenção Básica em Saúde: caminhos da institucionalização. Coordenação técnica: Instituto de Saúde Coletiva da Universidade Federal da Bahia e Instituto Materno Infantil Prof. Fernando Figueira (IMIP). Brasília, DF: Ministério da Saúde, 2005.

BRASIL. Ministério da Saúde. Diretrizes do NASF: Núcleo de Apoio a Saúde da Família. Brasília, DF: Ministério da Saúde, 2010.

BRASIL. Decreto n. 7.508 , de 28 de junho de 2011. Regulamenta a Lei n. 8.080, de 19 de setembro de 1990, para dispor sobre a organização do Sistema Único de Saúde - SUS, o planejamento da saúde, a assistência à saúde e a articulação inter federativa, e dá outras providências. Brasília, DF: Presidência da República, 2011a.

BRASIL. Ministério da Saúde. Portaria n. 2.488, de 21 de outubro de 2011. Aprova a Política Nacional de Atenção Básica, estabelecendo a revisão de diretrizes e normas para a organização da Atenção Básica, para a Estratégia Saúde da Família (ESF) e o Programa de Agentes Comunitários de Saúde (PACS). Brasília, DF: Ministério da Saúde, 2011b.

BRASIL. Agência Nacional de Saúde Suplementar. Dados gerais. Rio de Janeiro: Ministério da Saúde, 2012a.

BRASIL. Departamento de Atenção Básica. Atenção básica e a saúde da família. Brasília, DF: Ministério da Saúde, 2012b.

BRASIL. Departamento de Atenção Básica. Números. Brasília, DF: Ministério da Saúde, 
2012c.

CONTANDRIOPOULOS, A. et al. Avaliação na área de saúde: conceitos e métodos. Rio de Janeiro: Fiocruz/Hartz Z. A., 1997. p. 29-48.

COSTA, Glauce Dias da et al. Avaliação em saúde: reflexões inscritas no paradigma sanitário contemporâneo. Physis, Rio de Janeiro, v. 18, n. 4, p. 705-26, 2008.

DONABEDIAN, A. The quality of medical care. Science 200, 1978.

ESPERIDIÃO, Monique; TRAD, Leny Alves Bomfim. Avaliação de satisfação de usuários. Ciênc. Saúde Coletiva, Rio de Janeiro, 2005.

FELISBERTO, Eronildo. Monitoramento e avaliação na atenção básica: novos horizontes. Revista Brasileira de Saúde Materno Infantil, Recife, v. 4, n. 3, p. 317-21, 2004.

FREZ, Andersom Ricardo; NOBRE, Maria Inês Rubo de Souza. Satisfação dos usuários dos serviços ambulatoriais de fisioterapia da rede pública. Fisioter. Mov. (Impr.), Curitiba, v. 24, n. 3, set. 2011.

GOMES, R. et al. A atenção básica à saúde do homem sob a ótica do usuário: um estudo qualitativo em três serviços do Rio de Janeiro. Ciênc. Saúde Coletiva, v. 16, n. 11, p. 451321, 2011.

HARTZ, Zulmira M. A. Institucionalizar e qualificar a avaliação: outros desafios para a atenção básica. Ciência e Saúde Coletiva, v. 7, n. 3, p. 419-21, 2002.

MADUREIRA, Paulo Roberto de; DE CAPITANI, Eduardo Mello; CAMPOS, Gastão Wagner de Souza. Avaliação da qualidade da atenção à saúde na rede básica. Cad. Saúde Pública, Rio de Janeiro, v. 5, n. 1, jan. 1989.

McLAUGHLIN, J. A.; JORDAN, G. B. Logic models: a tool for telling your program's performance story. Evaluation and Program Planning, v. 2, p. 65-72, 1999.

MEDEIROS, Flávia A. et al. Acolhimento em uma Unidade Básica de Saúde: a satisfação do usuário em foco. Rev. Salud Pública, Bogotá, v. 12, n. 3, jun. 2010.

MEDINA, M. G. et al. Uso de modelos teóricos na avaliação em saúde: aspectos conceituais e operacionais. In: HARTZ, Z. A.; VIEIRA-DA-SILVA, L. M. (org.). Avaliação em saúde: dos modelos teóricos à prática na avaliação de programas e sistemas de saúde. Salvador: EDUFBA; Rio de Janeiro: Fiocruz, 2005.

MENDES, E. V. Uma agenda para a saúde. São Paulo: Hucitec, 1999.

MISHIMA, S. M. et al. A assistência na saúde da família sob a perspectiva dos usuários. Rev. Latino-Am. Enfermagem, Ribeirão Preto, v. 18, n. 3, jun. 2010.

PAIVA, Daniela Cristina Profitti de; BERSUSA, Ana Aparecida Sanches; ESCUDER, Maria Mercedes L. Avaliação da assistência ao paciente com diabetes e/ou hipertensão pelo Programa Saúde da Família do Município de Francisco Morato, São Paulo, Brasil. Cad. Saúde Pública, Rio de Janeiro, v. 22, n. 2, fev. 2006.

PATTON, M. Q. Utilization-focused evaluation. The new century text. Thousands Oaks, London, New Delhi: SAGE Puublications, 1997.

PINHEIRO, Roseni; MARTINS, Paulo Henrique Novaes. Avaliação em saúde na perspectiva do usuário: abordagem multicêntrica. Rio de Janeiro: CEPESC/IMS-UERJ; Recife: Editora Universitária UFPE; São Paulo: ABRASCO, 2011.

PUCCINI, Paulo de Tarso; CORNETTA, Vitória Kedy. Ocorrências em pronto-socorro: eventos sentinela para o monitoramento da atenção básica de saúde. Cad. Saúde Pública, Rio de Janeiro, v. 24, n. 9, set. 2008.

ROSA, R. Borba; PELEGRINI, A. H. Weis; LIMA, M. A. Dias da Silva. Resolutividade da assistência e satisfação de usuários da Estratégia de Saúde da Família. Rev. Gaúcha Enferm., Porto Alegre, v. 32, n. 2, p. 345-51, jun. 2011.

SANTOS, M. P. Avaliação da qualidade dos serviços públicos de atenção à saúde da criança sob a ótica do usuário. Revista Brasileira de Enfermagem, v. 48, p. 109-19, 1995.

SIMÉANT, S. Capacidad resolutiva de la demanda de atención de morbilidad a nivel primario. Bol Oficina Sanit Panam, v. 97, p. 114-25, 1984.

TINÔCO, Dinah dos Santos; SOUZA, L. M.; BARBOSA, Alba de Oliveira. Avaliação de políticas públicas: modelo tradicional e pluralista. Revista de Políticas Públicas (UFMA), v. 15, p. 
305-13, 2011.

TRAD, Leny Alves Bomfim et al. Estudo etnográfico da satisfação do usuário do Programa de Saúde da Família (PSF) na Bahia. Ciênc. Saúde Coletiva, São Paulo, v. 7, n. 3, 2002.

TRAVASSOS, Cláudia et al. Desigualdades geográficas e sociais na utilização de serviços de saúde no Brasil. Ciênc. Saúde Coletiva, Rio de Janeiro, v. 5, n. 1, 2000.

TURRINI, Ruth Natalia Teresa; LEBRÃO, Maria Lúcia; CESAR, Chester Luiz Galvão. Resolutividade dos serviços de saúde por inquérito domiciliar: percepção do usuário. Cad. Saúde Pública, Rio de Janeiro, v. 24, n. 3, mar. 2008.

VASCONCELOS, E. M. A priorização das famílias nas políticas de saúde. Revista de Atenção Primária à Saúde, Juiz de Fora, v. 2, n. 4, p. 20-8, 2000.

\section{Notas:}

1

De 2005 a 2012, a Unochapecó aprovou projetos em três editais do Programa Nacional de Reorientação da Formação Profissional em Saúde (Pró-Saúde) e em quatro editais do Programa de Educação pelo Trabalho (PET), estes com foco na Saúde da Família, Vigilância à Saúde e Saúde Mental.

\section{Lucimare Ferraz}

Enfermeira e Professora do Programa Stricto sensu em

Ciências da Saúde da Universidade Comunitária da Região de Chapecó - UNOCHAPECO

Doutora em Ciências Sociais pela Universidade Federal de

São Paulo - UNIFESP.

E-mail: Iferraz@unochapeco.edu.br

\section{Maria Elizabeth Kleba}

Enfermeira

Professora dos Programas Stricto sensu em Ciências da

Saúde e em Políticas Sociais e Dinâmicas Regionais da

Universidade Comunitária da Região de Chapecó - UNOCHA-

PECO

Doutora em Filosofia pela Universidade de Bremen-Alemanha

E-mail: Ikleba@unochapeco.edu.br

\section{Fátima Ferretti}

Fisioterapeuta.

Professora do Programa Stricto Sensu em Ciências da Saúde da Universidade Comunitária da Região de Chapecó - UNO-

CHAPECO.

Doutora em Ciências da Saúde pela Unifesp

E-mail: ferrettifisio@yahoo.com.br

\section{Natacha Luana Pezzuol Frank}

Acadêmica do curso de Medicina da Universidade Comunitária da Região de Chapecó - UNOCHAPECO.

E-mail: natachaluana@unochapeco.edu.br

\section{Universidade Comunitária da Região de Chapecó}

Av. Senador Attílio Fontana, 591 - E

Efapi - CEP: 89809-000, Chapecó - SC 